\title{
Modelo de la dinámica de neurotransmisores por medio de caminantes aleatorios
}

\author{
Jorge A. Herrera ${ }^{1}$, Juan C. Chimal ${ }^{1}$, Norma Sánchez Salas ${ }^{2}$ \\ 1 Instituto Politécnico Nacional, \\ Centro de Investigación en Computación, \\ Ciudad de México, México \\ 2 Instituto Politécnico Nacional, \\ Escuela Superior de Física y Matemáticas, \\ Ciudad de México, México \\ jaherrera@yahoo.com.mx, jchimale@gmail.com,norma@esfn.ipn.mx
}

\begin{abstract}
Resumen. En el presente artículo se propone modelar la dinámica de neurotransmisores por medio de caminantes aleatorios, se muestra que es posible afirmar que existe una relación de la distancia entre neuronas y la cantidad de neurotransmisores que llegan a los receptores de la neurona post-sináptica, con la finalidad de poder entender a grandes rasgos cómo funcionan las conexiones neuronales en términos de las sinápsis químicas. Concretamente, se comparan resultados experimentales de la implementación del modelo en donde se hace una cuantificación del número de moléculas que llegan a los receptores contra los resultados obtenidos por Leonid P. Savtchenko and Dmitri A. Rusakov [1] (corriente en función de la distancia).
\end{abstract}

Palabras clave: Automatas celulares, caminantes aleatorios, neurotransmisores.

\section{Model of the Dynamics of Neurotransmitters by Means of Random Walkers}

\begin{abstract}
In the present article we propose to model the dynamics of neurotransmitters by means of random walkers, it is possible to affirm that there is a relation of the distance between neurons and the amount of neurotransmitters that reach the receptors of the post-synaptic neuron, with The purpose of being able to understand roughly how neural connections work in terms of chemical synapses. Specifically, we compare experimental results of the implementation of the model where a quantification is made of the number of molecules that reach the receptors against the results o btained by LEONID P. SAVTChENKO AND DMITRI A. Rusakov [1] (current as a function of distance).
\end{abstract}

Keywords: Cellular automaton, random walkers, neurotransmitters. 


\section{Introducción}

Para poder entender cómo funcionan las conexiones neuronales, es necesario hablar sobre sinápsis química, misma que figura como una forma por medio de la cual las neuronas se comunican para poder enviar información e interactuar entre ellas $[11,12,13]$.

Aunque el sistema nervioso humano ha sido objeto de estudio a través de los siglos y dentro de diversas culturas [6], aún existen demasiadas preguntas en muy diversos campos relacionados al tema de las neuropatologías, que si bien en un punto determinado podría pensarse lo contrario, pueden estar relacionadas directamente con la manera en que se llevan a cabo conexiones neuronales de forma particular. Dentro de este conjunto de preguntas se encuentran muchas pertenecientes al estudio de enfermedades variadas como el Parkinson, Alzheimer, autismo, adicciones [2], la epilepsia [3,4], etc., las cuales que hoy en día no tienen cura y a su vez no se han terminado de entender.

Después de la publicación del modelo de Hodgkin y Huxley se ha abierto un panorama más amplio en cuanto al estudio de la dinámica neuronal desde el área de las matemáticas y con la ayuda de diversas técnicas de simulación y modelado; sobre todo en términos del papel que la química neuronal juega en la respuesta eléctrica de la misma, pues resultados apuntan a que estas investigaciones parecen tener muchas respuestas por brindar aún, de esta manera, los modelos discretos como el que se presenta en este artículo no son ni serán la excepción al intentar brindar no sólo respuestas, si no métodos más sencillos y computacionalmente menos costosos para realizar experimentación comparados con algunos modelos continúos, así mismo es viable creer que los modelos computacionales que se están desarrollando puedan derivar en nuevos protocolos de tratamiento [5], lo cual figura como un gran avance en el área de las ciencias biomédicas para estudio de neuropatologas particularmente.

\section{Antecedentes}

\subsection{Autómatas celulares}

Los automatas celulares son un tipo de modelos discretos, siendo estos una versión idealizada de un sistema complejo [8], estos están conformados por células o celdas dentro de un lattice, en donde a cada paso de tiempo las células pueden mostrar diversos estados, es decir, una célula individual dentro del lattice podría adoptar determinada condición dependiendo, principalmente, del estado (de la célula en cuestión) en $t_{n-1}$ y el estado de las células que están a su alrededor, mismas que conforman su vecindario finito.

De forma simple es posible nombrar a cada célula como $c_{i}$ y así mismo al autómata como un vector $\boldsymbol{C} \in \mathbb{Z}$, dentro del cual cada estado de alguna célula dada, en un tiempo dado $t_{i}$, podrá ser definido como $S_{i}$ contenido dentro de un conjunto $\boldsymbol{S} \in \mathbb{Z}$ de estados posibles para las células.

Por último se define la vecindad de una célula cualquiera en el autómata como $V_{i}=\left\{c_{1}, c_{2}, \ldots, c_{n}\right\}$, la cual puede ser de diversos tipos (según las condiciones 
del fenómeno que se desee modelar), de entre las más sencillas y conocidas se encuentran, por ejemplo, la vecindad de Vonn Neuman y la vecindad de Moore. A grandes rasgos la vecindad se refiere al conjunto contiguo de celdas y la posición respectiva entre cada una de ellas.

\subsection{Caminantes aleatorios y difusividad}

La difusión es tal vez el mecanismo más básico de transporte en la materia y se encuentra al origen de muchos procesos. Las caminatas aleatorias ofrecen un marco teórico útil para entender la difusión a un nivel micróscopico [9].

De forma simple, un caminante aleatorio es la forma discreta de un proceso difusivo, en el cual la probabilidad juega un papel importante a cada paso de tiempo discreto y en la trayectoria del caminante aleatorio, la cual está conformada por todos los puntos espaciales por donde el caminante pasa desde el tiempo inicial $t_{0}$ hasta el tiempo final $t_{n}$

Considérese que una particula es capaz de permanecer en posiciones discretas y equidistantes que se encuentran en una línea, dicha partícula tiene la posibilidad de moverse a lo largo de esas posiciones discretas en cada paso de tiempo discreto $t_{i}$ ya sea en dirección izquierda o derecha con igual probabilidad $\frac{1}{2}$, entonces la probabilidad $P_{t i}(p n)$ de que el caminante se encuentre en el sitio $p n$ al instante $t_{i}$ se define por medio de:

$$
P_{t i}(p n)=\frac{1}{2} P_{t i-1}(p n-1)+\frac{1}{2} P_{t i-1}(p n+1) .
$$

Asumiendo que el caminante se encuentra en la posición $p n=0$ en el tiempo inicial $t_{0}=0$ se puede describir la probabilidad de que el caminante haya dado $n$ pasos al tiempo $t_{i}$ a la derecha y $t_{i}-n$ pasos a la izquierda mediante la siguiente igualdad:

$$
P_{t i}(p n)=\left(\begin{array}{c}
t_{i} \\
n
\end{array}\right) \frac{1}{2^{t_{i}}} .
$$

Tomando en cuenta tiempos discretos grandes $(t>>1)$ y haciendo uso de la aproximación de Stirling, tenemos como resultado la distribución gaussiana:

$$
P_{t i}(p n)=\frac{1}{\sqrt{4 \pi D t}} e^{\frac{-n^{2}}{4 D t}}
$$

Considerando un tiempo continuo y una probabilidad igual de saltar hacía la derecha o izquierda $\alpha d t$ además de tomar un intervalo finito de tiempo $[t, t+d t]$ y una tasa $\alpha=1$ se puede escribir una ecuación para la evolución de $P_{t}(p n)$ :

$$
\frac{\partial P_{t}(p n)}{\partial t}=P_{t}(n p+1)-2 P_{t}(n p)+P_{t}(n p-1)
$$

Obteniendo la transformada de Fourier discreta de la expresión anterior se obtiene la siguiente ecuación:

$$
\frac{\partial P_{t}(p n)}{\partial t}=\left[e^{i} k+e^{-} i k-2\right] P(k, t) .
$$


Integrando la expresión anterior dadas las condiciones iniciales donde $P_{t=0}(n)$ $=\delta_{n, 0}$ implica que: $P(k, t=0)=1$ se puede obtener el siguiente resultado:

$$
P(k, t)=e^{2(\cos k-1) t} .
$$

El cual puede ser reducido mediante identidades trigonométricas y las funciones de Bessel dando como resultado:

$$
\frac{\partial P(x, t)}{\partial t}=D \frac{\partial^{2} P(x, t)}{\partial x^{2}} .
$$

Ahora bien, de aquí se puede deducir la expresión para el desplazamiento cuadrático medio $\left\langle x^{2}\right\rangle$ (segundo momento de la distribución $P(x, t)$ ) mediante la expresión siguiente, misma que muestra a $\left\langle x^{2}\right\rangle$ con una tendencia de crecimiento con el paso del tiempo:

$$
\left\langle x^{2}\right\rangle(t)=\int_{-\infty}^{\infty} x^{2} P(x, t), d x=2 D t
$$

Tomando en cuenta una suma de posiciones que contiene $\mathrm{N}$ pasos:

$$
x=\sum_{n p=1}^{N} u_{n} .
$$

Con $u_{n}$ generados a partir de una distribución de probabilidad $p(u)$ obtenemos (1) en una forma general:

$$
P(x, N)=\int_{-\infty}^{\infty} P\left(x^{\prime}, N-1\right) p\left(x-x^{\prime}\right), d x^{\prime} .
$$

La ecuación anterior puede resolverse mediante la simplificación de la misma por transformada de Fourier y considerando algunas condiciones iniciales de las cuales, se sabe que:

$$
P(x, N)=\frac{1}{\sqrt{2 \pi N\left\langle u^{2}\right\rangle}} e^{-\frac{x^{2}}{2 N\left\langle u^{2}\right\rangle}}
$$

Sustituyendo x por un vector posición r, la expresión anterior puede generalizarse a espacios d-dimensionales, como en la siguiente expresión:

$$
P(r, t)=\frac{1}{(4 \pi D t)^{d / 2}} e^{-\frac{r^{2}}{4 D t}}
$$

de donde se obtiene:

$$
\left\langle r^{2}\right\rangle=2 d D t
$$




\section{Modelo propuesto}

Al ser liberados los neurotransmisores viajan por el espacio sináptico de forma tal que avanzan hacía los receptores mediante un movimiento aleatorio, dada esta condición se propone modelar la dinámica de las moléculas por medio de caminantes aleatorios.

El autómata celular fue implementado a través de código escrito en Matlabß, las gráficas de los resultados obtenidos por medio de experimentación computacional de la cantidad de neurotransmisores que llegan a los receptores en función de la distancia entre las dendritas tienen una forma similar a los resultados de experimentación en [1].

\subsection{Lattice}

Se modeló el lattice mediante una matriz de $2 x n$ la cual contiene los límites de este, y la información sobre la posición ocupada por una célula dada. Por consiguiente, los estados de cada célula dentro del lattice simplemente se reducen a las siguientes condiciones: un espacio $(x, y)$ dentro del lattice ocupado por una célula (célula viva) será registrado en la matriz $v_{c a}$, por consiguiente un espacio descoupado no será tomado en cuenta en los registros de coordenadas de la matriz $v_{c a}$ (como célula viva) y por tanto no desempeñará ningún papel en la dinámica del modelo.

El lattice ha sido pensado como un medio espacial, y las posiciones de las dendritas toman el papel de límites en el medio sináptico de tal forma que las moléculas no pueden moverse, por ejemplo, más allá de la posición $x=d$ de la dendrita post-sináptica ni hacía atras de la posición en $x=d_{0}$ de la dendrita pre-sináptica. Es decir, las posiciones en $x$ de las dendritas, juegan un papel de barrera para las células, antes y después de los cuales las moleculas no podrán hacer movimiento alguno a menos que estas logren alcanzar una posición $y=R$ (veáse Fig. 1) determinada, más abajo o más arriba de los parametros propuestos en el programa determinados como en [1], o bien alcanzen la posición $(d, y)$ de un receptor.

Es importante señalar que el modelo del lattice no permite que dos o más células $c_{i}$ del autómata ocupen un mismo lugar (coordenada) $(x, y)$ dentro del lattice definido por la matriz $v_{c a}$ para asegurar dicha condición se lleva un proceso de control el cual permite que una célula $c_{i}$ conozca su vecindad y tenga conocimiento sobre las posibilidades de movimiento que tiene.

\subsection{Movimiento}

Seguidas de las consideraciones sobre el lattice, se puntuarán aquellas relacionadas con el movimiento. De forma particular, se hablará sobre cómo las células dentro del lattice eligen una "dirección" hacía la cual avanzar en cada instante de tiempo $t_{i}$.

Dado que el fenómeno a modelar sigue un comportamiento de movimiento aleatorio 2-dimensional, el programa se apegará esta dinámica de las células en 


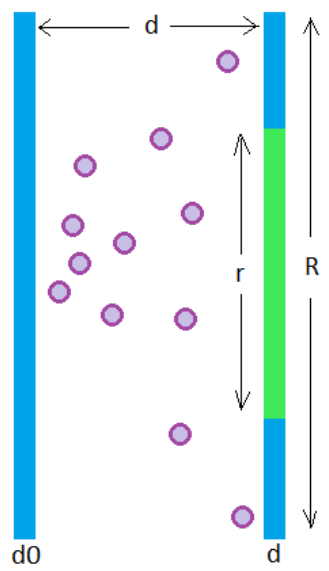

Fig. 1. Representación gráfica del modelo, particularmente el lattice, el cual se extiende desde $d_{0}$ hasta $d$ y toda la longitud de $R$, de la misma manera que en [1] el modelo presentado en este trabajo tiene una zona activa (representada por el área verde), los puntos morados representan a las células del autómata y por último $d$ es la distancia entre las dendritas de las neuronas (distancia de hendidura sináptica).

el autómata mediante un vecindario del tipo Von Neuman, esto implica que las células pueden tener 4 posibilidades de movimiento: arriba, abajo, derecha e izquierda, y la dirección será elegida mediante una gestión de probabilidad, para ello será necesario hacer uso de números pseudoaleatorios generados por la funcion $\operatorname{rand}()$ de Matlabß, dicho proceso se verá de forma más detalla más adelante.

En resumen, el algoritmo procede de la siguiente manera: genera un número aleatorio entre 0 y 1 denominado $J$, si $J<=0.25$ entonces la célula en el autómata $c_{i}$ se moverá hacía arriba pero si $0.25<J<=0.5 c_{i}$ se moverá una posición abajo o, dado $0.5<J<=0.75 c_{i}$ se desplazará hacía la derecha o bien si $0.75<J<=1 c_{i}$ tendrá un desplazamiento hacía la izquierda.

\subsection{Receptores}

En la barrera de la dendrita post-sináptica encontraremos los receptores, mismos que están distribuidos a lo largo de una región activa como en [1]. Los receptores a grandes rasgos son tomados como coladeras en el modelo (veáse Fig. 2 ), y tienen una posición $(d, y)$, donde $d$ es la distancia a la cual se encuentra la dendrita post-sináptica.

El papel desempeñado por los receptores es el de absorber a las células que se encuentren en sus mismas posiciones $(d, y)$, entonces, aunque los caminantes (células) del autómata lleguen al límite espacial $d$ si estos no coinciden estrictamente con alguna posición $(d, y)$ de un receptor en particular, seguirán 


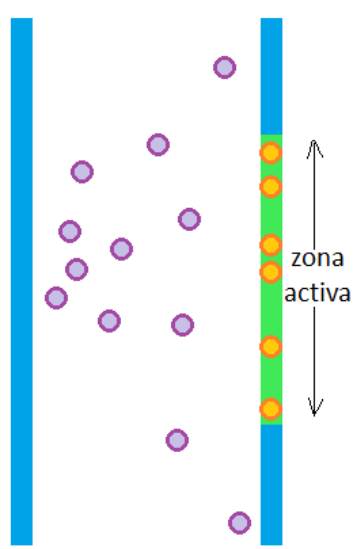

Fig. 2. Representación gráfica de cómo está considerado el límite derecho de lattice (dendrita post-sináptica), el cual contiene receptores distribuidos aleatoriamente a lo largo de la zona activa (zona verde) a la espera de que alguna molécula (puntos morados) llegué a alguna posición $(d, y)$ para ser absorbida.

moviéndose a través de la hendidura y por consecuencia no formarán parte del conteo de células absorbidas por el receptor.

Es importante mencionar que todos los receptores tienen diferentes coordenadas en $y$ lo cual implica directamente que no será posible que dos receptores se encuentren en la misma posición $(d, y)$ a lo largo de la ejecución del programa, así mismo el algoritmo se encarga de hacer que estos estén distribuidos de manera aleatoria y normal a lo largo de la zona activa.

Por último, es importante mencionar que el tamaño de la zona activa es una variable fundamental en el desempeño de la tarea de los receptores en el algoritmo ya que las variaciones más simples del tamaño de esta genera resultados totalmente diferentes.

\subsection{Pseudocódigo}

En términos generales el pseudocódigo presentado en este apartado da una idea general de cómo funciona el programa, además de que mediante el mismo es posible hacer no sólo modificaciones al momento de realizar simulación, sino también nuevas implementaciones a lo largo del mismo con el objetivo de tener al alcance la posibilidad hacer diversos tipos de experimentación según sean requeridos. 
Jorge A. Herrera, Juan C. Chimal, Norma Sánchez Salas

Pseudocódigo para la Simulación de la dinámica de Neurotransmisores:

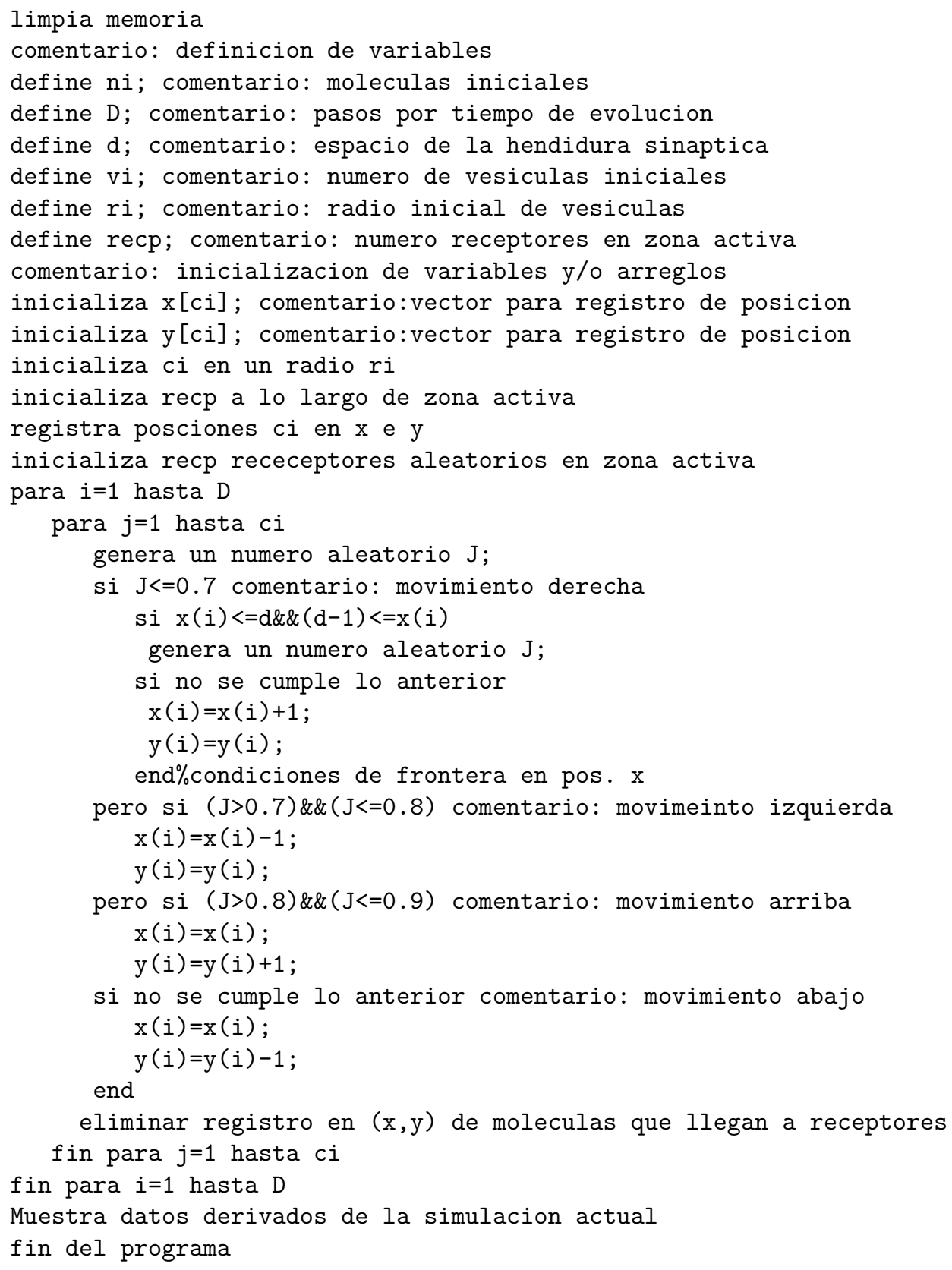

En el pseudocódigo se encuentra una sentencia de control if-else (dentro de la gestión de $J$ para el movimiento hacía la derecha) encargada de dar las condiciones de frontera, a grandes rasgos esta condición está pensada para evitar que los caminantes pasen más allá de la dendrita post-sináptica si es que en el 
tiempo $t_{i}$ no han alcanzado una altura (posición $y$ ) suficiente, entonces y como se mencionó anteriormente, la dendrita post-sináptica se modeló como si fuese un muro con algunos huecos (los cuales juegan el papel de receptores) distribuidos a lo largo de una zona activa como en el modelo de [1] y con diferentes posiciones.

Una vez que se cumpla la condición en donde una o más células son absorbidas por los receptores, los registros de las mismas serán eliminados de la matriz $v_{c a}$, esto implica que tendrá menos elementos en la siguiente entrada de los bucles y las moleculas absorbidas no desempearán ningún papel en la dinámica del algoritmo, es decir, serán eliminadas por completo de los registros en el programa y pasarán a ser estrictamente parte de la cuantificación para los resultados del mismo.

\section{Experimentos y resultados}

A continuación se abordará lo relacionado a los datos obtenidos mediante la experimentación con el modelo ya implementado en Matlabß, comenzaremos presentando los resultados obtenidos por [1], En los cuales, se puede apreciar en las gráficas una función de la corriente respecto de la distancia.

En general estos datos demuestran que el pico de corriente corriente estará en función de la separación entre dendritas, además de que habrá un intervalo donde la corriente será máxima; por consiguiente también habrá intervalos donde la amplitud de corriente decaiga.

El modelo que se propone en este trabajo muestra que al momento de hacer una cuantificación de los neurotransmisores que llegan a los receptores postsinpticos también se obtendrán curvas con formas muy similares a las de [1].
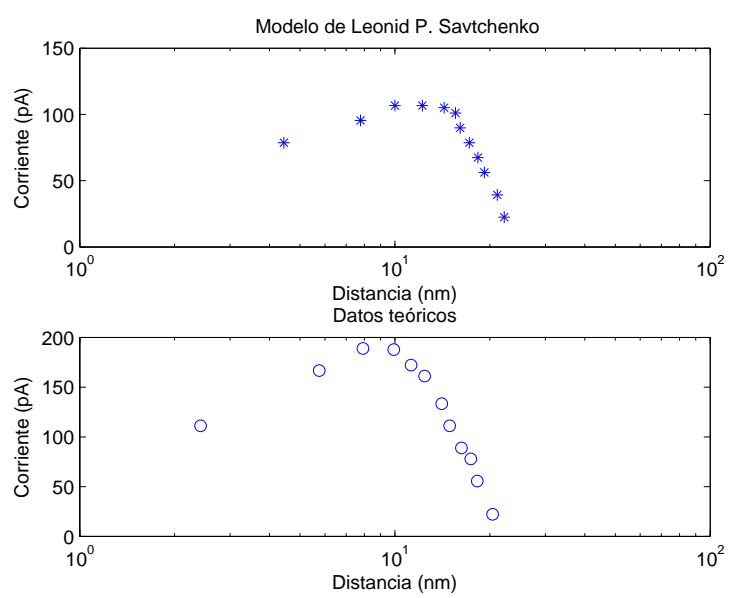

Fig. 3. Datos obtenidos por [1] mediante su modelo propuesto comparados con datos teóricos. 
Jorge A. Herrera, Juan C. Chimal, Norma Sánchez Salas
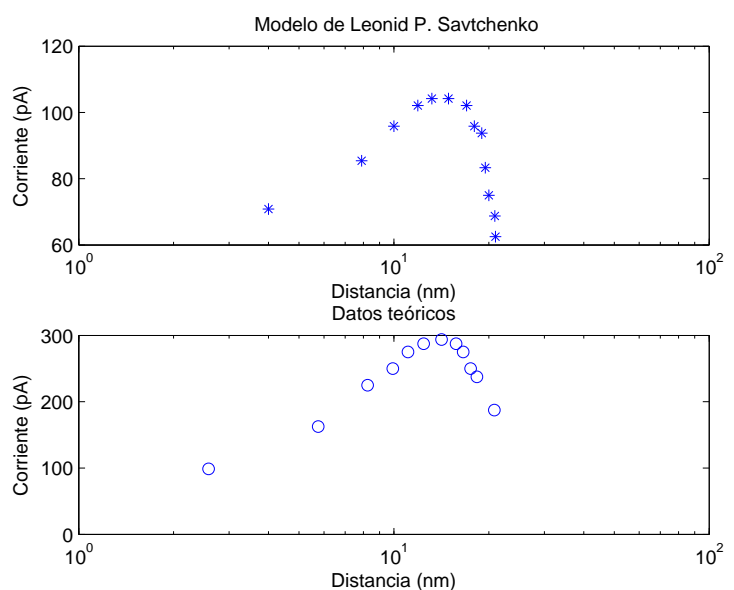

Fig. 4. Datos obtenidos por [1] mediante su modelo propuesto comparados con datos teóricos.

Por otro lado, se presentan los resultados obtenidos por medio de experimentación con el modelo aquí propuesto, en el cual se encontrarán gráficas en donde el número de células que llegan a los receptores está en función de la distancia de separación entre dendritas.

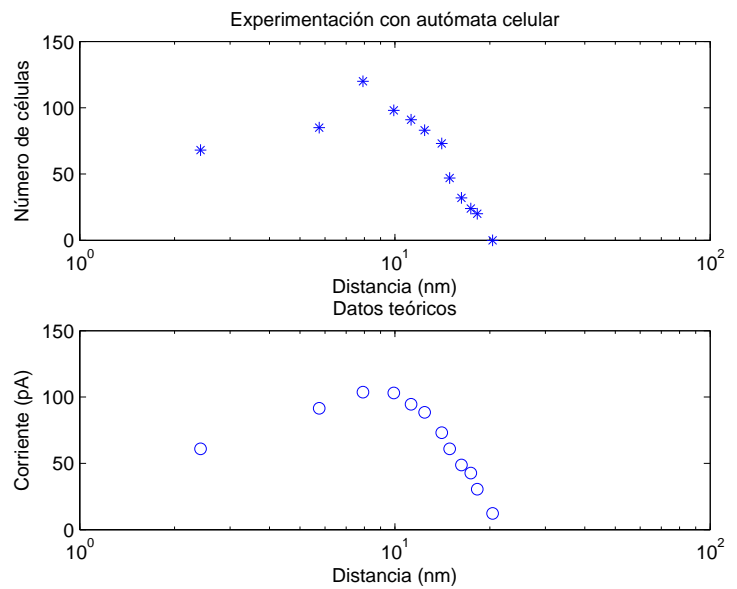

Fig. 5. Datos obtenidos por el modelo propues comparados contra datos teóricos. 

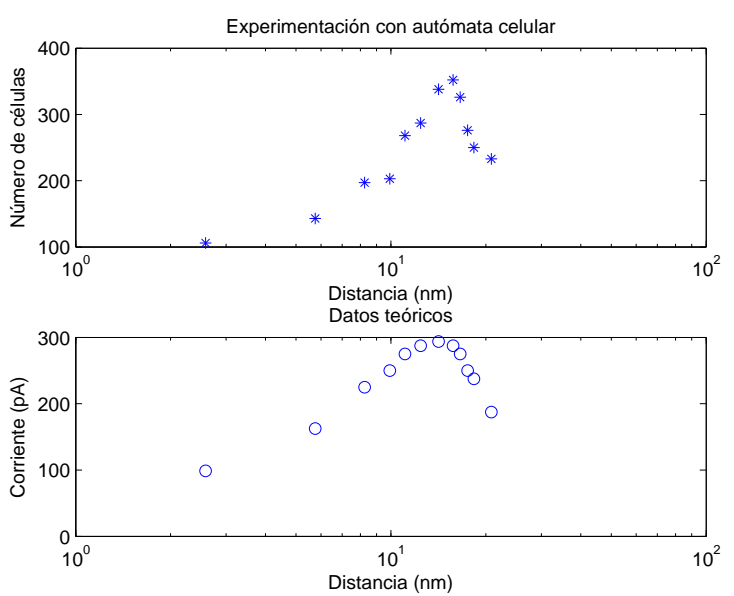

Fig. 6. Datos obtenidos por el modelo propuesto comparados contra datos teóricos.

Estos últimos datos que se presentan en las gráficas anteriores (Fig 5. y Fig 6) y que se obtuvieron a través de la experimentación muestran que aún para la cantidad de moleculas de neurotransmisores que llegan a los receptores post-sinápticos es importante tomar en cuenta la distancia de separación entre dendritas, los datos presentados anteriormente tienen una característica importante: al igual que en las gráficas para los datos del modelo de [1] existe una distancia a la cual el número de moléculas de neurotransmisores que llegan a los receptores es máximo.

Lo anterior bien puede apuntar hacía una relación en la que se podría mostrar que la distancia y el número de moleculas en los receptores juegan un papel determinante en el disparo de corriente producido en una sinápsis.

\section{Trabajo futuro}

La presente investigación tiene posibilidades abiertas para que se desempeñen más experimentaciones, dentro de las que podrían resaltarse principalmente aquellas que puedan ampliar el modelo y/o generalizarlo, por ejemplo, a una modelación de una serie de neuronas liberando neurotransmisores entre sí.

Por otro lado también queda abierto el estudio sobre la relación entre la concentración de moleculas que llegan a la neurona post-sináptica y el pico de corriente producido por los mismos, de hecho, es viable formar una función que pueda describir este fenómeno a partir de los datos generados por el autómata.

Así mismo, el modelo ofrece la posibilidad de hacer experimentación con la respuesta eléctrica obtenida mediante diversos parámetros en el autómata, o agregados a este, con la finalidad de poder estudiar diferentes dinámicas y, derivadas de estas, diferentes respuestas eléctricas. 
Computacionalmente existen también posibilidades para trabajo futuro, sobre todo en el tema de optimización del código el cual podra ser llevado a cabo a través el uso de computo paralelo que ofrece la toolbox dedicada de Matlabß, implementando el algoritmo en algún otro lenguaje o bien hacer uso de la arquitectura CUDA (en algún equipo que lo permita); esto principalmente con la finalidad de realizar pruebas más complejas sobre todo en cuanto al manejo de un mayor número de células en el autómata celular en un menor tiempo de ejecución.

\section{Discusión y conclusiones}

Los resultados de las experimentaciones muestran que la cantidad de moléculas que llegan a los receptores en la dendrita post-sináptica está directamente relacionada con la separación entre las dendritas (tamaño de hendidura sináptica).

Se logró modelar y simular computacionalmente la dinámica de las moleculas de los neurotransmisores dando como resultado una comparación de datos exitosa, lo cual implica que la implementación del modelo funciona de acuerdo a lo que se esperaba, aunque cabe mencionar que las experimentaciones previas apuntan que el modelo bien podría ser adaptado para modelar un fenómeno sináptico más complejo donde, por ejemplo, un número inicial de vesiculas $>1$ con radios iguales o diferentes sean las nuevas condiciones iniciales.

Esta investigación derivó en un nuevo modelo discreto mediante el cual es posible hacer experimentación con los parámetros dados y propuestos de manera sencilla y rápida. Además el modelo propuesto está totalmente abierto a cambios con todas y cada una de las variables, lo cual implica que esta flexibilidad al momento de hacer modificaciones pueden permitir modelar no sólo sinápsis más complejas, si no también experimentos donde a este proceso se le puedan agregar perturbaciones las cuales apunten a intentar responder preguntas específicamente sobre enfermedades, que estén relacionadas con procesos neuroquímicos (más específicamente sinápsis) anómalos, ahorrando tiempo y brindando una herramienta de experimentación confiable.

Agradecimientos. Agradecemos a Germán Téllez del CIC-IPN por los comentarios y correcciones hechas a la sección teórica de este artículo.

\section{Referencias}

1. Savtchenko, L.P., Rusakov, D.A.: The optimal height of the synaptic cleft. PNAS, 104, pp. 1823-1828 (2006)

2. Spronsen, M.V., Hoogenraad, C.C.: Synapse Pathology in Psychiatric and Neurologic Disease Current. Neurology and Neuroscience Reports, 10(4), pp. 207-214 (2010) 
3. Badawya, R.A.B., Harveyc, A.S., Macdonella, R.A.L.: Cortical hyperexcitability and epileptogenesis: Understanding the mechanisms of epilepsy - Part 1. Journal of Clinical Neuroscience, 16, pp. 355-36 (2009)

4. Badawya, R.A.B., Harveyc, A.S., Macdonella, R.A.L.: Cortical hyperexcitability and epileptogenesis: Understanding the mechanisms of epilepsy - Part 2. Journal of Clinical Neuroscience, 16, pp. 485-500 (2009)

5. Stefanescu, R.A., Shivakeshavan, R.G., Talathi, S.S.: Computational models of epilepsy. Seizure, 21, pp. 748-759 (2012)

6. Carod-Artal, F.J., Vzquez-Cabrera, C.B.: Paleopatologa neurolgica en las culturas precolombinas de la costa y el altiplano andino (II). Historia de las trepanaciones craneales, Rev Neurol. 38, pp. 886-894 (2004)

7. Bernard, C.: Alterations in synaptic function in epilepsy. https://www.ncbi.nlm. nih.gov/books/NBK98161/

8. Mitchel, M.: Complexity A Guided Tour, Oxford University press (2009)

9. Boyer, D.: Fronteras de la Física en el Siglo XXI. Procesos difusivos: de moléculas a animales, UNAM, México (2013)

10. Jonas, P., Major, G., Sakmann, B.: Quantal components of unitary EPSCs at the mossy fibre synapse on CA3 pyramidal cells of rat hippocampus. J Physiol, 472, pp. 615-663 (1993)

11. Purves, D., Augustine, G.J., Fitzpatrick, D.: Neuroscience, 2nd edition. Sunderland (MA), Sinauer Associates (2001)

12. Neurons, Synapses, Action Potentials, and Neurotransmission http://www . mind. ilstu.edu/curriculum/neurons_intro/neurons_intro.php

13. Thiele, S., Nash, J.: Chemical Synapses. http://www.els.net/WileyCDA/ ElsArticle/refId-a0000037.html

14. Gilat, A.: MATLAB: Una introducción con ejemplos prácticos, Reverte (2006)

15. Strogatz, S.H.: Nonlinear Dynamics And Chaos: With Applications To Physics, Biology, Chemistry, and Engineering (Studies in Nonlinearity) 1st edition, AddisonWesley (1994)

16. Weisstein, E.W.: Elementary Cellular Automaton. http://mathworld.wolfram. com/ElementaryCellularAutomaton.html

17. Rangel-Mondragon, J.: A Catalog of Cellular Automata. http://library. wolfram.com/infocenter/MathSource/505/ 